\title{
SEDE DO PACIENTE CIRÚRGICO ORTOPÉDICO NO PÓS-OPERATÓRIO IMEDIATO
}

\author{
THIRST OF THE ORTHOPEDIC SURGICAL \\ PATIENT IN THE IMMEDIATE POSTOPERATIVE
}

\section{SED DEL PACIENTE QUIRÚRGICO ORTOPÉDICO EN EL POSTOPERATORIO INMEDIATO}

\author{
Rayane Nascimbeni Maldonado \\ Marília Ferrari Conchon ${ }^{2}$ \\ Marcela Maria Birolim ${ }^{3}$ \\ Ligia Fahl Fonseca ${ }^{4}$
}

Como citar este artigo: Maldonado RN, Conchon MF, Birolim MM, Fonseca LF. Sede do paciente cirúrgico ortopédico no pós-operatório imediato. Rev baiana enferm. 2020;34:e34533.

\begin{abstract}
Objetivo: verificar a prevalência, intensidade e desconforto da sede do paciente cirúrgico ortopédico e as correlações com as variáveis demográficas e clínico-cirúrgicas. Método: estudo transversal, quantitativo e analítico com 78 pacientes em Sala de Recuperação Pós-Anestésica de um hospital de ensino. Resultados: oito pacientes (10,3\%) verbalizaram sede espontaneamente. Sua intensidade média foi de 4,8 e o desconforto de 5,0. O atributo boca seca foi o mais prevalente, seguido de vontade de beber água e lábios ressecados. Intensidade associou-se significativamente a desconforto da sede no grupo de pacientes de 21 a 40 anos. Houve relação de prevalência entre presença de sede e todos os atributos da Escala de Desconforto da Sede Perioperatória, com exceção do atributo gosto ruim na boca. Conclusão: a sede do paciente cirúrgico ortopédico é prevalente no período pós-operatório, de intensidade moderada, porém consideravelmente desconfortável.
\end{abstract}

Descritores: Sede. Enfermagem Perioperatória. Ortopedia. Sala de Recuperação.

Objective: to assess the prevalence, intensity and discomfort of the thirst of the orthopedic surgical patient and the correlations with the demographic and clinical-surgical variables. Method: cross-sectional, quantitative and analytical study with 78 patients in the Post-Anesthetic Recovery Room of a teaching hospital. Results: eight patients (10.3\%) reported thirst spontaneously. Its mean intensity was 4.8, and the discomfort, 5.0. The attribute dry mouth was the most prevalent, followed by desire to drink water and dried lips. Intensity correlated significantly with discomfort of thirst in the group of patients from 21 to 40 years. There was a relationship of prevalence between presence of thirst and all the attributes of the Perioperative Thirst Discomfort Scale, with the exception of the attribute bad taste in the mouth. Conclusion: the thirst of the orthopedic surgical patient is prevalent in the postoperative period, with moderate intensity, but considerably uncomfortable.

Descriptors: Thirst. Perioperative Nursing. Orthopedics. Recovery Room.

Objetivo: evaluar la prevalencia, la intensidad y la incomodidad de la sed del paciente ortopédico quirúrgico y las correlaciones con las variables demográficas y clinico-quirúrgicas. Método: estudio transversal, cuantitativo y analítico con 78 pacientes en la Sala de Recuperación Post-Anestésica de un hospital universitario. Resultados: ocho pacientes (10,3\%) reportaron sed espontáneamente. Su intensidad media fue de 4,8 y la incomodidad, 5, 0. El

\footnotetext{
Enfermeira. Mestre em Enfermagem. Pesquisadora Independente. Londrina, Paraná, Brasil. rayane_nascimbeni@hotmail.com. http://orcid.org/0000-0002-23446983.

Enfermeira. Doutora em Enfermagem. Professora da Universidade Estadual de Londrina. Londrina, Paraná, Brasil. http://orcid.org/0000-0003-0393- I559.

Enfermeira. Doutora em Enfermagem. Professora da Faculdade Guairacá. Guarapuava, Paraná, Brasil. http://orcid.org/0000-000 I-69764955.

Enfermeira. Doutora em Enfermagem. Professora Associada da Universidade Estadual de Londrina. Londrina, Paraná, Brasil. http://orcid.org/0000-000 I-7550-9/4I .
} 
atributo boca seca fue el más frecuente, seguido por la voluntad de beber agua y labios secos. Intensidad correlacionó significativamente al malestar de la sed en el grupo de pacientes de 21 a 40 años. Hubo una relación de prevalencia entre la presencia de la sed y todos los atributos de la Escala de Incomodidad de la Sed Perioperatória, con la excepción del atributo mal sabor en la boca. Conclusión: la sede del paciente ortopédico quirúrgico es frecuente en el postoperatorio, de intensidad moderada, pero muy incómodo.

Descriptores: Sed. Enfermería Perioperatória. Ortopedia. Sala de Recuperación.

\section{Introdução}

O procedimento cirúrgico é uma forma terapêutica utilizada pela medicina no tratamento de uma variedade de distúrbios fisiopatológicos Não obstante, pode implicar ameaças reais, como risco de vida, perda de órgãos ou parte destes, mudanças na rotina, afastamento da família e de compromissos profissionais, prejuízos financeiros, além do desconforto decorrente da própria intervenção cirúrgica ${ }^{(1-2)}$.

Dentre os preparos físicos do paciente no período pré-operatório, destaca-se o jejum, que provoca fome, ansiedade, repercussões hormonais e, particularmente, é um dos fatores predisponentes da sede. Outras condições também podem intensificar a sede no perioperatório, como intubação orotraqueal, utilização de opioides e anticolinérgicos e fatores emocionais ${ }^{(3)}$.

Os procedimentos cirúrgicos ortopédicos representam uma grande porcentagem das agendas cirúrgicas de diversas instituições hospitalares. Em uma instituição referência para ortopedia e traumatologia, foram realizados, em 2016, 7.285 procedimentos cirúrgicos, dos quais $1.466(17,8 \%)$ eram ortopédicos, totalizando uma média de 123 cirurgias ortopédicas por mês. Por outro lado, destaca-se a alta taxa de suspensão cirúrgica dessa clínica, em torno de 26\% em 2013, sendo a segunda especialidade com maior número de suspensão ${ }^{(4)}$. Este evento contribui para o prolongamento da internação hospitalar e a repetição de preparos pré-operatórios.

O paciente cirúrgico ortopédico apresenta peculiaridades que contribuem para um estado de grande desconforto e ansiedade, como mobilidade prejudicada e maior risco para o desenvolvimento de infecções, que podem ser relacionadas à realização de múltiplos procedimentos e à utilização de órteses e próteses que impedem ou dificultam sua mobilização ${ }^{(5)}$, somados à dor, que é bastante prevalente nesse grupo de pacientes ${ }^{(6)}$.

Desse modo, destaca-se a relação entre ansiedade e sede. Fatores emocionais podem ser responsáveis pelo declínio da produção salivar, ocasionando a sensação de boca seca $^{(7)}$, um dos atributos mais prevalentes entre pacientes cirúrgicos no pós-operatório imediato (POI), atingindo $87,3 \%{ }^{(8)}$.

O desencadeamento da sede no paciente cirúrgico ortopédico pode ainda decorrer da utilização de medicamentos da classe dos opiáceos, utilizados no controle da dor pós-operatória ${ }^{(6)}$. Assim como os anticolinérgicos, os opioides provocam a diminuição da produção salivar como um efeito adverso, ocasionando, por consequência, o ressecamento da cavidade oral ${ }^{(9)}$.

O objetivo deste artigo é verificar a prevalência, intensidade e desconforto da sede do paciente cirúrgico ortopédico e as correlações com as variáveis demográficas e clínico-cirúrgicas.

\section{Método}

Trata-se de um estudo transversal de abordagem quantitativa e analítica, que permite descrever a situação e as relações entre os fenômenos de interesse em um momento ${ }^{(10)}$.

Esta pesquisa foi realizada na Sala de Recuperação Anestésica (SRA) do Centro Cirúrgico de um hospital de ensino no Sul do Brasil. Trata-se de um hospital-escola público, com 316 leitos e atendimento exclusivo para o Sistema Único de Saúde (SUS), referência municipal e estadual nos casos de alta complexidade e traumatologia.

A população do estudo foi composta por pacientes no POI de cirurgias ortopédicas. 
A amostra não probabilística foi constituída por pacientes submetidos a procedimentos cirúrgicos ortopédicos entre novembro de 2016 e março de 2017. Foram incluídos pacientes que atenderam aos seguintes critérios: idade igual ou superior a 12 anos, jejum de 2 horas ou mais, permanecer na SRA ou em Sala Operatória (SO) após a realização do procedimento cirúrgico, estar consciente e orientado quanto ao tempo e espaço. Foram excluídos aqueles com dificuldade auditiva ou de comunicação.

Para a coleta de dados, utilizou-se um roteiro de pesquisa com dados demográficos e clínicos. A coleta ocorreu na SRA e, para pacientes que se recuperavam em sala cirúrgica, por precaução de contato ou por aguardar vaga de Unidade de Terapia Intensiva (UTI), realizou-se a coleta na SO.

A intensidade da sede foi mensurada por meio da Escala Visual Numérica (EVN), que consiste em um instrumento unidimensional com graduação de 10 pontos (0-10), onde zero é classificado como nenhuma sede e 10, a maior sede já sentida $^{(9)}$. O desconforto da sede foi mensurado pela Escala de Desconforto da Sede Perioperatória (EDESP), com validação de conteúdo e fidedignidade de 100\% para todos os itens, a qual avalia sete atributos da sede: boca seca, lábios ressecados, língua grossa, saliva grossa, garganta seca, gosto ruim na boca e vontade de beber água. Sua graduação varia de 0 a 14, em que 0 significa nenhum desconforto e 14, extremamente desconfortável ${ }^{(11)}$.

Usou-se planilha eletrônica Excel $2014{ }^{\circledR}$ para a construção do banco de dados. Estes foram inseridos por dupla digitação, seguida de validação para corrigir possíveis erros na introdução das informações, com posterior exportação para o programa IBM SPSS versão 20.0. Primeiramente as variáveis foram analisadas de forma descritiva, por meio de frequências absolutas e relativas. Para as análises de correlação, foram consideradas variáveis preditoras: sexo, idade, tempo de jejum de líquidos e sólidos, ASA, duração da anestesia, intubação, utilização de opioide, tempo de procedimento, intensidade da sede e os atributos da EDESP. Para a análise da correlação das variáveis preditoras com a intensidade e o desconforto da sede pós-operatória, foi utilizado o coeficiente de correlação de Spearman. Para a correlação entre presença de sede e variáveis preditoras, usou-se o coeficiente de correlação de Pearson (r), com intervalo de confiança de 95\%.

A pesquisa teve aprovação do Comitê de Ética em Pesquisa, CAAE 29069414.5.0000.5231, e respeitou os princípios éticos da Resolução n. 466, de novembro de 2012, do Conselho Nacional de Saúde. Todos os participantes, após orientações e esclarecimentos sobre o estudo, assinaram o Termo de Consentimento Livre Esclarecido (TCLE). Os responsáveis por pacientes com idade inferior a 18 anos assinaram o Termo de Assentimento Livre e Esclarecido (TALE).

\section{Resultados}

Durante o período do estudo, foram realizados 558 procedimentos cirúrgicos ortopédicos, totalizando o atendimento de 488 pacientes, dos quais $248(50,8 \%)$ eram eletivos. Da totalidade de procedimentos, 247 (44,3\%) eram eletivos e 311 (55,7\%) de caráter de urgência/emergência. A diferença entre os números de procedimentos e pacientes deveu-se ao fato de ter-se realizado mais de um procedimento cirúrgico em um mesmo indivíduo.

A amostra deste estudo foi constituída de 78 pacientes, a maioria do sexo masculino (74,4\%), com idade entre 12 e 85 anos ( $\mu$ 42,8 \pm DP 20,2). Destes 78 pacientes, 62 apresentaram sede. O tempo de jejum de líquidos variou entre 3,8 e 23,9 horas ( $\mu$ 14,0 \pm DP 3,9); já o tempo de jejum de sólidos variou entre 9,0 e 24,3 horas ( $\mu 15,5 \pm 3,6$ ).

Quanto à suspensão cirúrgica, 23 pacientes $(29,5 \%)$ tiveram o procedimento cirúrgico cancelado pelo menos uma vez: para 18 deles $(23,1 \%)$, suspensão de um procedimento cirúrgico, para $4(5,1 \%)$, suspensão de dois, e 1 paciente $(1,3 \%)$ teve três suspensões cirúrgicas. Sobre os motivos das suspensões cirúrgicas, de acordo com classificação própria da instituição, dentre 11 causas de cancelamento verificadas neste estudo, $5(45,4 \%)$ estavam relacionadas à solicitação da equipe. Dez suspensões relacionavam-se com 
atraso da cirurgia anterior (31,3\%); outras oito, à recusa do cirurgião (25,0\%); quatro, ao atendimento de urgência/emergência (12,5\%); e três, à programação cirúrgica para o dia errado $(9,4 \%)$. Os motivos preparo inadequado pelo médico, preparo inadequado pelo paciente, falta de vaga de UTI, duplicação de agendamento, paciente sem condições clínicas, reagendado pela clínica e ausência do cirurgião foram relatados uma vez, representando 3,1\% cada.

Em relação à classificação do estado físico do paciente no pré-operatório, de acordo com a American Society of Anesthesiologists ${ }^{(12)}$, a maioria dos pacientes foi classificada como ASA I (44,9\%), saudáveis, e ASA II (47,4\%), paciente com doença sistêmica leve ou moderada, sem limitação funcional. Os demais foram classificados como ASA III (5,1\%), paciente com doença sistêmica severa com limitação funcional; e ASA IV (1,3\%), paciente com doença sistêmica severa que representa risco de vida constante. Um paciente não foi classificado pela escala da ASA, por ter sido submetido a anestesia local para a realização do procedimento cirúrgico.

Bloqueios raquidiano e peridural foram utilizados na maioria dos procedimentos $(55,1 \%)$, seguidos dos bloqueios de membros superiores $(19,2 \%)$ e de anestesia geral (11,5\%). Apenas três pacientes $(3,8 \%)$ foram submetidos à sedação, e oito (10,3\%) tiveram associações de anestesia: raquianestesia com geral, geral com peridural, bloqueio de membros superiores com geral, sedação mais raquianestesia e ainda outro foi submetido apenas à anestesia local.

A maior parte dos pacientes $(83,3 \%)$ não foi intubada no intraoperatório. Quanto ao uso de opioides, o fentanil foi utilizado em 25 pacientes (32,1\%), a morfina em 13 (16,7\%), e 32 (41,0\%) receberam a associação desses dois opioides. Em 6 pacientes $(7,7 \%)$ foram utilizados outros opioides ou outras combinações desses medicamentos: alfentanil, sulfentanil associado ao fentanil, alfentanil combinado com fentanil. Em 2 pacientes (2,6\%) não foram utilizados opiáceos.

Os procedimentos cirúrgicos foram realizados predominantemente nos membros inferiores $(65,4 \%)$, seguindo-se cirurgias de membros superiores (29,5\%) e de coluna (5,1\%).

Em relação à verbalização espontânea de sede, oito pacientes (10,3\%) relataram estar com sede. Dentre os pacientes que não verbalizaram estar com sede espontaneamente (89,7\%), após questionamento pelos pesquisadores, $54(77,1 \%)$ expressaram verbalmente estar com sede. A média de intensidade da sede no POI foi de 4,8 ( \pm DP 0,7) (Gráfico 1).

Gráfico 1 - Distribuição do percentual da intensidade da sede conforme a Escala Visual Numérica por número de pacientes. Londrina, Paraná, Brasil - 2017

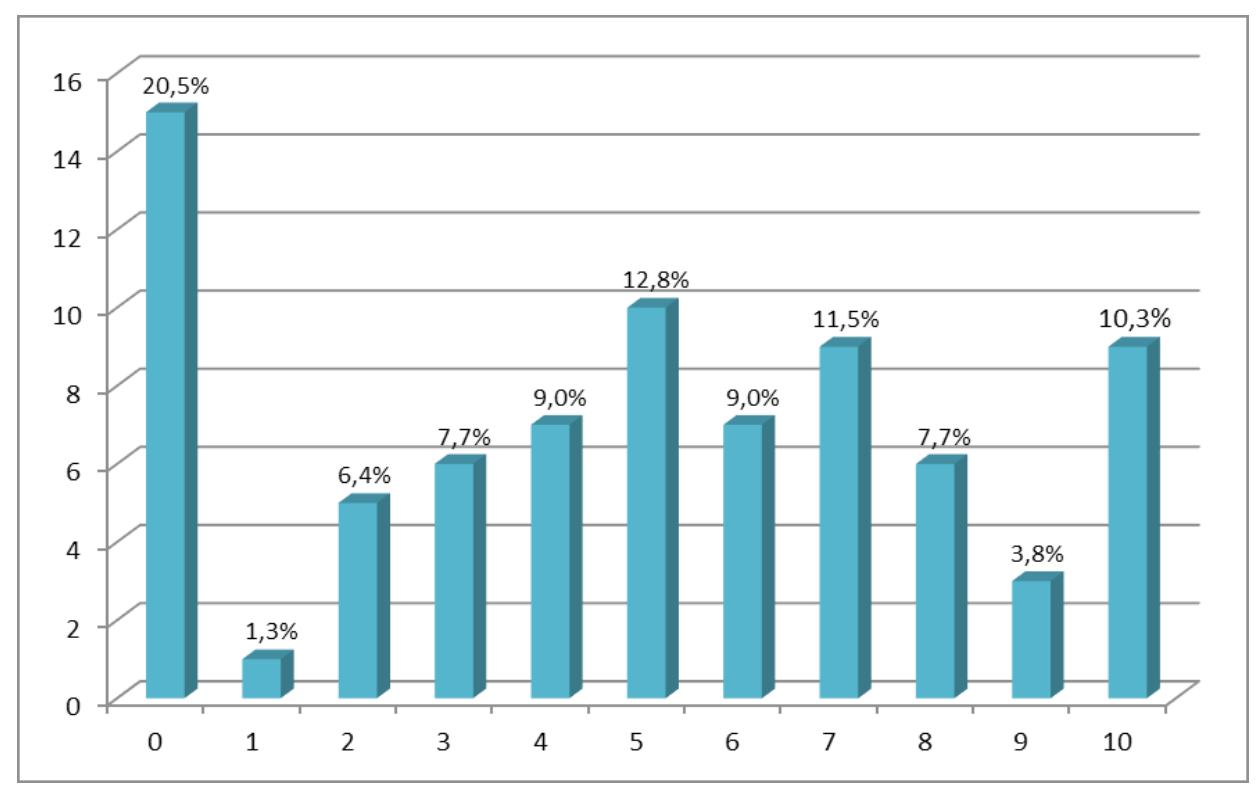

Fonte: Elaboração própria 
O escore médio de desconforto mensurado pela EDESP foi 5,0 ( \pm DP 3,4). A análise da prevalência dos atributos da escala separadamente, mostrou que o atributo boca seca foi o de maior prevalência $(80,8 \%)$, seguido de vontade de beber água (79,5\%) e lábios ressecados $(71,7 \%)$.
Entretanto, quanto à graduação dos atributos (nada, pouco e muito), vontade de beber água apresentou maior prevalência de muito desconfortável (23,1\%), seguido de lábios ressecados $(17,9 \%)$, gosto ruim na boca $(17,9 \%)$ e boca seca (15,4\%) (Gráfico 2).

Gráfico 2 - Distribuição do percentual dos atributos de desconforto da sede por número de pacientes. Londrina, Paraná, Brasil - 2017

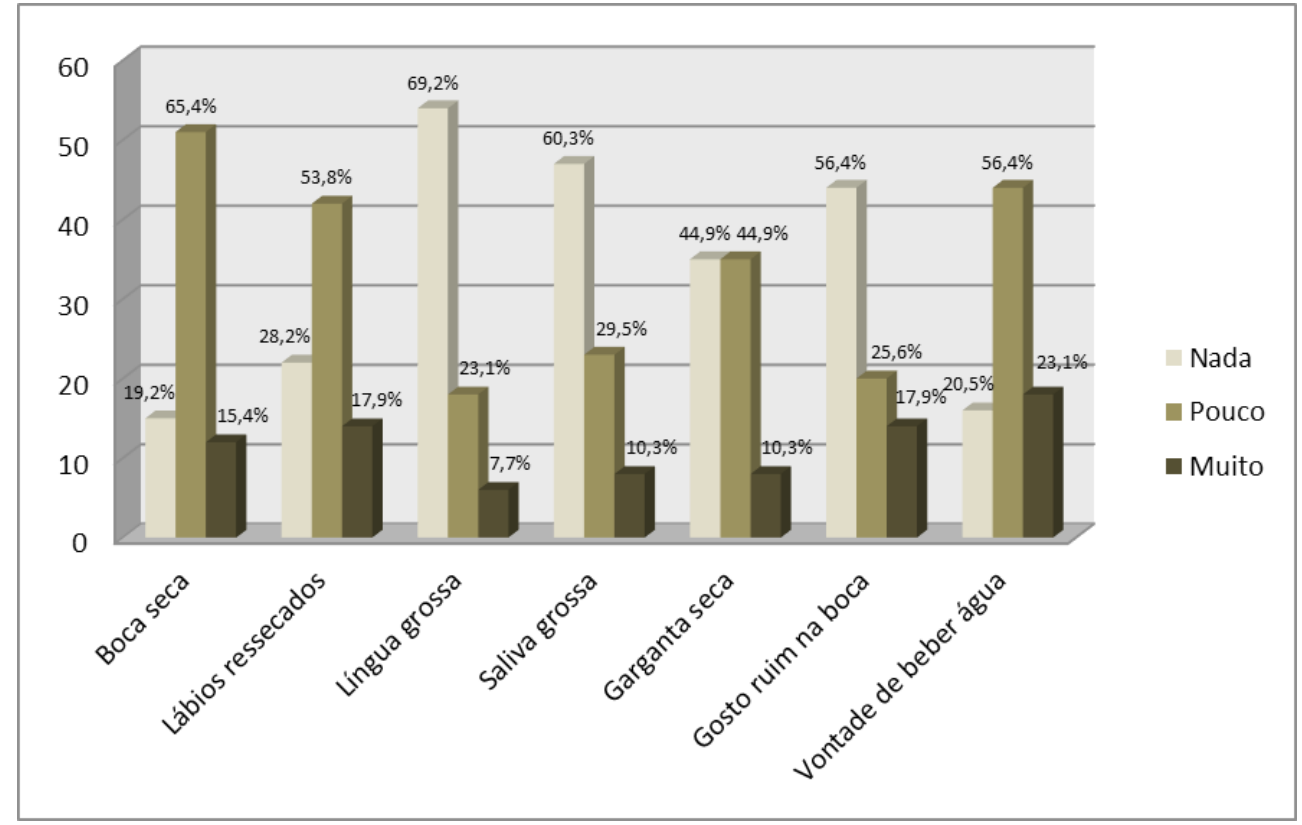

Fonte: Elaboração própria.

Não foi encontrada associação significativa na análise bivariada entre variáveis independentes (idade, tempo de jejum de líquidos, tempo de jejum de sólidos, duração da anestesia, tempo de procedimento) e variáveis dependentes (intensidade e desconforto da sede). Verificou-se, porém, que a intensidade da sede no POI apresentou associação significativa $(\mathrm{p}=0,000)$ com o desconforto da sede, isto é, quanto maior a intensidade da sede referida pelo paciente, maior foi o desconforto da sede perioperatória indicado pela EDESP (Tabela 1).

Tabela 1 - Correlações das variáveis preditoras com as pontuações da Escala Visual Numérica em pacientes submetidos a cirurgia ortopédica no pós-operatório imediato. Londrina, Paraná, Brasil - 2017

\begin{tabular}{l|c|c|c|c}
\hline \multirow{2}{*}{ Variáveis } & \multicolumn{2}{|c|}{ Desconforto da Sede } & \multicolumn{2}{c}{ Intensidade da Sede } \\
\cline { 2 - 5 } & $\mathbf{r}^{*}$ & p-valor & $\mathbf{r}^{*}$ & p-valor \\
\hline Idade & $-0,098$ & 0,392 & $-0,045$ & 0,695 \\
Tempo de jejum de líquidos & 0,058 & 0,617 & 0,006 & 0,960 \\
Tempo de jejum de sólidos & 0,073 & 0,525 & 0,130 & 0,258 \\
Duração da anestesia & $-0,131$ & 0,251 & $-0,201$ & 0,078 \\
Tempo do procedimento & 0,076 & 0,508 & $-0,078$ & 0,497 \\
Intensidade da sede & 0,633 & 0,000 & 1,00 & - \\
\hline
\end{tabular}

Fonte: Elaboração própria.

* r - coeficiente de correlação de Spearman. 
Os fatores associados à presença de sede foram: idade entre 21 e 40 anos, boca seca, lábios ressecados, língua grossa, saliva grossa e garganta seca. As variáveis sexo, idade até
20 anos e 41 anos e mais, ASA, intubação e utilização de opioides e o atributo gosto ruim na boca não apresentaram relação de prevalência significativa (Tabela 2).

Tabela 2 - Distribuição das variáveis demográficas e clínicas de acordo com a presença de sede em pacientes submetidos a cirurgia ortopédica no pós-operatório imediato. Londrina, Paraná, Brasil - 2017

\begin{tabular}{l|c|c|c|c}
\hline \multirow{2}{*}{ Variáveis } & \multicolumn{4}{|c}{ Presença de Sede } \\
\cline { 2 - 5 } & n (\%) & $\begin{array}{c}\text { Relação de } \\
\text { Prevalência }\end{array}$ & $\begin{array}{c}\text { Intervalo de } \\
\text { Confiança }\end{array}$ & p-valor \\
\hline
\end{tabular}

\section{Sexo}

\begin{tabular}{|c|c|c|c|}
\hline Feminino & $17(85,0)$ & 1,09 & $0,87-1,38$ \\
\hline Masculino & $45(77,6)$ & 1,00 & \\
\hline
\end{tabular}

\section{Idade}

\begin{tabular}{|c|c|c|c|c|}
\hline Até 20 anos & $11(73,3)$ & 1,02 & $0,71-1,47$ & 0,909 \\
\hline De 21 a 40 anos & $23(95,8)$ & 1,33 & $1,08-1,65$ & 0,008 \\
\hline 41 anos e mais & $28(71,8)$ & 1,00 & & \\
\hline \multicolumn{5}{|l|}{ ASA } \\
\hline I & $30(85,7)$ & 1,00 & & \\
\hline II & $32(76,2)$ & 0,89 & $0,72-1,10$ & 0,286 \\
\hline \multicolumn{5}{|l|}{ Intubação } \\
\hline $\operatorname{Sim}$ & $9(69,2)$ & 0,85 & $0,580-1,24$ & 0,399 \\
\hline Não & $53(81,5)$ & 1,00 & & \\
\hline \multicolumn{5}{|c|}{ Utilização de opioide } \\
\hline Sim & $61(80,3)$ & 1,60 & $0,40-6,44$ & 0,505 \\
\hline Não & $1(50,0)$ & 1,00 & & \\
\hline \multicolumn{5}{|l|}{ Boca Seca } \\
\hline Sim & $55(87,3)$ & 1,87 & $1,08-3,24$ & 0,025 \\
\hline Não & $7(46,7)$ & 1,00 & & \\
\hline \multicolumn{5}{|l|}{ Lábios Ressecados } \\
\hline Sim & $49(87,5)$ & 1,48 & $1,03-2,13$ & 0,033 \\
\hline Não & $13(59,1)$ & 1,00 & & \\
\hline \multicolumn{5}{|l|}{ Língua Grossa } \\
\hline Sim & $23(95,8)$ & 1,33 & $1,10-1,60$ & 0,003 \\
\hline Não & $39(72,2)$ & 1,00 & & \\
\hline \multicolumn{5}{|l|}{ Saliva Grossa } \\
\hline Sim & $29(93,5)$ & 1,33 & $1,08-1,64$ & 0,007 \\
\hline Não & $33(70,2)$ & 1,00 & & \\
\hline \multicolumn{5}{|l|}{ Garganta Seca } \\
\hline Sim & $38(88,4)$ & 1,29 & $1,01-1,65$ & 0,046 \\
\hline Não & $24(68,6)$ & 1,00 & & \\
\hline
\end{tabular}

Fonte: Elaboração própria.

* O atributo gosto ruim na boca não teve relação de prevalência. 


\section{Discussão}

Um acontecimento inusitado, desconhecido, quase sempre gera sentimentos de medo e ansiedade $^{(2)}$. Partindo desse pressuposto, uma cirurgia pode intensificar essas sensações negativas, quando se consideram as possíveis implicações de um procedimento cirúrgico no cotidiano do paciente, desde o afastamento da família e de compromissos profissionais até uma ameaça real à sua vida ${ }^{(1)}$. Uma cirurgia envolve uma série de cuidados pré-operatórios para sua realização, como o jejum, que, por sua vez, pode provocar, dentre outros sintomas e desconfortos, a sede.

Estudos demonstraram que a sede é um sintoma altamente prevalente e intenso ${ }^{(8,13)}$, não obstante, ainda é tema pouco explorado na literatura, principalmente aquela relacionada a pacientes cirúrgicos no pré e pós-operatório ${ }^{(3,7)}$. O cenário apresenta uma interface ainda pior, quando se analisa a sede e suas correlações em grupos específicos. Este estudo contribui com a análise da prevalência da sede e seus desconfortos em um grupo de pacientes cirúrgicos com características importantes para o desenvolvimento da sede perioperatória.

A relevância de pesquisar o sintoma sede neste grupo específico deve-se às peculiaridades desses pacientes na instituição do estudo, neste caso, um hospital de ensino com residência médica de ortopedia e traumatologia, sendo referência para trauma. Tais características podem contribuir para um perfil de serviço com fragilidades decorrentes de grande demanda. Dentre elas, longos períodos de espera para a realização do procedimento, tempo de internação prolongado, altas taxas de suspensão cirúrgica e, consequentemente, repetidos e prolongados períodos de jejum pré-operatório.

Observa-se, neste estudo, que os procedimentos cirúrgicos ortopédicos foram realizados predominantemente em indivíduos jovens do sexo masculino. Embora não se tenha investigado o motivo para a realização do procedimento cirúrgico, as características levam a crer que se relacione com causas externas, mais especificamente acidentes de trânsito. Na cidade onde se realizou o estudo, o número de acidentes de trânsito, entre 1999 e 2008, aumentou 74,2\%, e indivíduos do sexo masculino representavam mais de $70 \%$ das vítimas. Além disso, 60\% dos envolvidos nesses acidentes tinham entre 15 e $34 \operatorname{anos}^{(14)}$. Os dados demográficos desta pesquisa assemelham-se aos de outro estudo, em que o sexo masculino representa mais de $70 \%$ dos indivíduos envolvidos em acidentes de trânsito ${ }^{(15)}$.

A recomendação de tempo de jejum é de 2 horas para líquidos claros e de 6 horas para uma dieta leve ${ }^{(12)}$, porém o tempo de jejum pré-operatório verificado nesta pesquisa foi bastante superior, tendo média de 14 horas para líquidos e de 15,5 horas para sólidos, assemelhando-se a resultados obtidos por outros pesquisadores ${ }^{(8,13)}$. Em outro estudo realizado na mesma instituição desta pesquisa, o tempo de jejum máximo encontrado foi de 37 horas $^{(13)}$. Achados assim demonstram que os protocolos de preparo pré-operatório não são realizados, o que pode intensificar os desconfortos decorrentes do jejum - como a sede - e, sobretudo, influenciar na recuperação pós-operatória, ocasionando a potencialização da resposta orgânica ao trauma cirúrgico ${ }^{(16)}$.

A maioria dos pacientes foi classificada como ASA I e ASA II, isto é, eram indivíduos saudáveis ou com doenças sistêmicas leves, sem limitações $^{(12)}$. Essa característica pode estar relacionada à predominância de indivíduos jovens submetidos a procedimento cirúrgico em razão de trauma decorrente de acidente de trânsito.

Em relação à técnica anestésica, a raquianestesia/peridural e o bloqueio de membros superiores foram as mais frequentes. Pacientes vítimas de acidente de trânsito sofrem lesões principalmente em membros ${ }^{(17)}$. Nesta pesquisa, os procedimentos cirúrgicos foram realizados sobretudo em membros inferiores, por isso a predominância da raquianestesia/peridural.

A sede é sintoma comum entre pacientes cirúrgicos, porém pouco verbalizado pela maioria dos indivíduos ${ }^{(3)}$. Nesta pesquisa, a verbalização espontânea da sede foi de 10,3\%. Entretanto, depois de questionados os pacientes, esse número aumentou expressivamente $(77,1 \%)$. Observa-se, portanto, que, apesar da alta prevalência do 
sintoma, poucos pacientes a expressam verbalmente aos profissionais de saúde. Isso pode estar vinculado a uma cultura de que sentir sede é um preço a pagar para se ter segurança no procedimento anestésico-cirúrgico, impedindo complicações intra e pós-operatórias ${ }^{(18)}$.

Acredita-se que o silêncio esteja relacionado com o medo de falar sobre o real sentimento quanto ao desconforto. O silêncio do paciente cirúrgico não significa não ter sede; apenas retrata a fragmentada interação e comunicação entre paciente e profissional. É habitual o profissional de saúde questionar o paciente cirúrgico sobre presença de dor, desconsiderando desconfortos tão prevalentes como a sede $e^{(13)}$.

Num estudo em que o paciente atribuía valores monetários fictícios à prevenção dos desconfortos pós-operatórios, a sede foi o segundo desconforto com maior atribuição monetária, destacando-se como o segundo desconforto pelo qual os pacientes estavam dispostos a pagar mais para não vivenciá-lo ${ }^{(19)}$. Importa salientar que o paciente cirúrgico considera a sede extremamente desconfortável e estressora, mas se mantém calado por considerar que nada poderá ser feito para aliviar sua sede. Ao mesmo tempo, o silêncio do paciente cirúrgico quanto à presença e ao desconforto da sede provoca a intensificação do sintoma ${ }^{(18)}$, gerando maior sofrimento e angústia.

No presente estudo, alguns indivíduos apresentaram dificuldade de graduar a intensidade da sede que sentiam. Assim como a dor, a sede é um sintoma subjetivo, podendo ter variação de um indivíduo para outro em situação semelhante $^{(3)}$. Esta variação da percepção da sede pode estar relacionada com fatores fisiológicos - como a diferença da capacidade de organismos diferentes detectarem alterações na osmolaridade plasmática - e, sobretudo, com questões de hábitos pessoais ${ }^{(7-8,20)}$.

A sede pode ser definida como o desejo de beber água, porém a ingesta hídrica pode estar vinculada a outros fatores, como rituais, hábitos e desejo por outras substâncias ${ }^{(20)}$. Pode-se dizer, portanto, que a sede é uma sensação que pode ser aprendida ou condicionada a um processo que pode ser percebido por sinais e sintomas representados pelo organismo, como boca e garganta secas $^{(11)}$. Corroborando outros estudos, encontrou-se, neste estudo, que a sede, além de ser um sintoma de alta prevalência, é também de alta intensidade ${ }^{(8,13)}$.

Tendo em vista que a sede tem causa multifatorial e pode ser intensificada por fatores associados à internação e ao procedimento cirúrgico, é relevante não apenas mensurar sua intensidade, mas também considerar sua complexidade de sinais ${ }^{(11)}$. A sede pode ser identificada por outros meios, como aumento da osmolaridade, redução da pressão arterial e do volume sanguíneo, aumento da concentração da angiotensina $\mathrm{II}^{(7)}$. Também apresenta atributos indicativos de ressecamento de mucosa que levam a alterações no hormônio antidiurético (ADH) e na percepção da sede ${ }^{(21)}$.

Quanto aos atributos da EDESP, todos foram mencionados pelos pacientes nesta pesquisa, assemelhando-se ao resultado de um estudo em que boca seca e vontade de beber água também foram os itens de maior prevalência $(87,3 \%)^{(8)}$. Em outros estudos, esses atributos aparecem como sinais e sintomas decorrentes da sede ${ }^{(20)}$, observados tanto pelos profissionais quanto pelos próprios pacientes e seus familiares ${ }^{(22)}$.

O escore final da EDESP, nesta pesquisa, apresentou desconforto médio inferior ao resultado de outra pesquisa ${ }^{(8)}$. Mesmo quando resulta em desconforto de moderada intensidade, o sintoma é real, não pode ser ignorado, tampouco ficar sem receber estratégias de alívio.

Quanto à suspensão cirúrgica, verificou-se que, como em outros estudos, a especialidade ortopédica tem alta taxa de cancelamento ${ }^{(4,23)}$. Além disso, os principais motivos de suspensão cirúrgica não estão relacionados a fatores clínicos dos pacientes. Em outra pesquisa, o cancelamento a pedido do cirurgião representou $17,93 \%$ das suspensões ${ }^{(24)}$. O cancelamento cirúrgico, além de gerar prejuízos financeiros para a instituição, afeta em especial o paciente, que é submetido ao preparo cirúrgico repetidamente, com tempos extremos de jejum, podendo intensificar o desconforto da sede. 
Semelhantemente a outros estudos ${ }^{(3,8)}$, apesar do elevado tempo de jejum pré-operatório, não foi encontrada associação significativa desta variável com intensidade e desconforto da sede. Considera-se necessária a investigação da correlação destas variáveis em grupos estratificados e com tempo de jejum inferior, o que poderia identificar a correlação significativa entre tempo de jejum, intensidade e desconforto da sede em uma população com características demográficas semelhantes.

Quanto à duração da anestesia e tempo de procedimento, não se encontrou associação significativa entre intensidade e desconforto da sede. Neste sentido, acredita-se na hipótese de que alguns fatores possam influenciar os resultados, como mensuração não rigorosa de infusão de líquidos e falta de controle de sangramento intraoperatório.

Houve relação de prevalência significativa entre faixa etária de 21 a 40 anos e presença de sede. Essa relação entre idade e presença de sede precisa ser melhor investigada, com pesquisas que proporcionem a estratificação da amostra, permitindo observar e relacionar o comportamento das diversas faixas etárias com as demais variáveis em um modelo multifatorial.

Quanto à falta de relação de prevalência significativa com o uso de opioides, acredita-se que a dose-dependência pode influenciar na presença e intensidade da sede, que necessita ser melhor investigada ${ }^{(9)}$.

Foi possível observar relação de prevalência com presença de sede entre os pacientes que referiram boca seca, lábios ressecados, língua grossa, saliva grossa e garganta seca. Além disso, verificou-se correlação entre desconforto e intensidade, isto é, quanto maior o desconforto da sede perioperatória, maior a intensidade do sintoma, resultado semelhante ao de outro estudo ${ }^{(8)}$.

Diante da alta prevalência da sede, ressalta-se a importância da valorização deste sintoma por meio de sua identificação, mensuração e tratamento. Os profissionais têm subsídios disponíveis na literatura para utilizar em sua prática, como o Protocolo de Segurança para o Manejo da Sede (PSMS) ${ }^{(25)}$, que avalia as condições do paciente, para que receba estratégias de alívio da sede e de seus desconfortos.

Espera-se que os resultados evidenciados neste estudo contribuam para a melhoria do cuidado dos pacientes submetidos a procedimentos cirúrgicos, considerando a sede como um desconforto real e passível de tratamento no POI, permitindo um atendimento mais humanizado.

Acredita-se que a amostra não probabilística tenha sido a limitação do estudo, tendo em vista que esta foi delimitada por um período determinado, podendo interferir nos resultados encontrados.

\section{Conclusão}

Verificou-se que a sede é prevalente entre os pacientes cirúrgicos ortopédicos no POI, ainda que poucos a verbalizem espontaneamente. Houve correlação significativa entre intensidade e desconforto, isto é, quanto mais intensa a sede, maior o desconforto causado por ela.

Quanto à presença de sede, observou-se relação de prevalência de pacientes com idade entre 21 e 40 anos com atributos da EDESP - com exceção de gosto ruim na boca -, demonstrando que a sede é um sintoma que causa desconfortos.

Tendo em vista a alta prevalência e intensidade da sede, torna-se importante reconhecer este sintoma como agente estressor e desconfortável para o paciente cirúrgico, assim como a dor. A valorização deste sintoma pelos profissionais é indispensável para a identificação, mensuração e tratamento da sede.

\section{Colaborações:}

1 - concepção, projeto, análise e interpretação dos dados: Rayane Nascimbeni Maldonado, Marcela Maria Birolim e Lígia Fahl Fonseca;

2 - redação do artigo e revisão crítica relevante do conteúdo intelectual: Rayane Nascimbeni Maldonado, Marília Ferrari Conchon e Lígia Fahl Fonseca;

3 - aprovação final da versão a ser publicada: Rayane Nascimbeni Maldonado, Marília Ferrari Conchon e Lígia Fahl Fonseca. 


\section{Referências}

1. Santos CCA, Polgrossi JEF, Maia LFSM. Estresse do paciente frente ao cancelamento do procedimento cirúrgico. Rev Remecs [Internet]. 2018 [cited 2020 Jan 29];3(4):12-20. Available from: https://www. revistaremecs.recien.com.br/index.php/remecs/ article/view/21/pdf

2. de Juan K. O impacto da cirurgia e os aspectos psicológicos do paciente: uma revisão. Psicol Hosp (São Paulo) [Internet]. 2007 [cited 2018 Dec 9];5(1):48-59. Available from: http:// pepsic.bvsalud.org/scielo.php?script $=$ sci_ arttext\&pid $=$ S1677-74092007000100004\&lng=pt\&n $\mathrm{rm}=\mathrm{iso}$

3. Nascimento LA, Garcia AKA, Conchon MF, Aroni P, Pierotti I, Martins PR, et al. Advances in the management of perioperative patient's thirst. AORN J. 2020;111(2):165-79. DOI: 10.1002/ aorn.12931

4. Nascimento LA, Tillvitz LR, Fonseca LF. Suspensão cirúrgica: o ângulo estatístico de um problema de repercussões humanas. Rev enferm UFPE on line. 2013 nov;7(esp):6592-600. DOI: 10.5205/ reuol.5058-41233-3-SM.0711esp201305

5. Silva FS, Viana MF, Volpato MP. Diagnósticos de enfermagem em pacientes internados pela clínica ortopédica em um unidade médico-cirúrgica. Rev Gaúcha Enferm [Internet]. 2008 dez [cited 2017 Oct 27];29(4):565-72. Available from: http://www.seer. ufrgs.br/index.php/RevistaGauchadeEnfermagem/ article/view/3826/6542

6. Silva EJGDG, Dixe MACR. Prevalência e características da dor em pacientes internados em hospital português. Rev dor. 2013 dez;14(4):245-50. DOI: http://dx.doi.org/10.1590/ S1806-00132013000400003

7. Arai S, Stotts N, Puntillo K. Thirst in critically ill patients: from physiology to sensation. Am J Crit Care. 2013 Jul;22(4):328-35. DOI: 10.4037/ ajcc 2013533

8. Pierotti I, Fracarolli IL, Fonseca LF, Aroni P. Avaliação da intensidade e desconforto da sede perioperatória. Esc Anna Nery. 2018;22(3):e20170375. DOI: http://dx.doi.org/10.1590/2177-9465-ean-20170375

9. Stotts NA, Arai SR, Cooper BA, Nelson JE, Puntillo KA. Predictors of thirst in intensive care unit patients. J Pain Symptom Manage. 2015 Mar;49(3):530-8. DOI: $10.1016 /$ j.jpainsymman.2014.07.001
10. Polit DF, Beck CT. Fundamentos da pesquisa em enfermagem: avaliação de evidências para a prática de enfermagem. 7a ed. Porto Alegre: ArtMed; 2011.

11. Martins PR, Fonseca LF, Rosseto EG, Mai LD. Developing and validating the Perioperative Thirst Discomfort Scale. Rev Esc Enferm USP. 2017 Jul;51:e03240. DOI: 10.1590/S1980220X2016029003240

12. American Society of Anesthesiologists. Practice guidelines for preoperative fasting and the use of pharmacologic agents to reduce the risk of pulmonary aspiration: application to healthy patients undergoing elective procedures: an updated report by the American Society of Anesthesiologists Task Force on Preoperative Fasting and the Use of Pharmacologic Agents to Reduce the Risk of Pulmonary Aspiration. Anesthesiology. 2017 Mar;126(3):376-93. DOI: 10.1097/ALN.0000000000001452

13. Aroni P, Nascimento LA, Fonseca LF. Avaliação de estratégias no manejo da sede na sala de recuperação pós-anestésica. Acta paul enferm. 2012;25(4):530-6. DOI: http://dx.doi.org/10.1590/ S0103-21002012000400008

14. Teixeira RJ, Calixto R, Pinheiro ACD. O reflexo da "Lei Seca" nos acidentes de trânsito em Londrina. In: Peixe BCS. Formulação e gestão de políticas no Paraná: reflexões, experiências e contribuições. Curitiba: Escola de Governo do Paraná; 2010. v.2, p. $459-75$.

15. Gonsaga RAT, Rimoli CF, Pires EA, Zogheib FS, Fujino MVT, Cunha MB. Avaliação da mortalidade por causas externas. Rev Col Bras Cir. 2012;39(4)263-7. DOI: https://doi.org/10.1590/ S0100-69912012000400004

16. Oliveira KGB, Balsan M, Oliveira SS, AguilarNascimento JE. A abreviação do jejum préoperatório para duas horas com carboidrato aumenta o risco anestésico? Rev Bras Anestesiol. 2009;59(5):577-84. DOI: https://doi.org/10.1590/ S0034-70942009000500006

17. Simoneti FS, Cunha LO, Gurfinkel Y, Mancilha TS, Campioni FC, Cabral AH, et al. Padrão de vítimas e lesões no trauma de motocicletas. Rev Fac Ciênc Méd [Internet]. 2015 nov [cited 2017 Oct 22];18(1):3640. Available from: https://revistas.pucsp.br/index. php/RFCMS/article/viewFile/24711/pdf

18. Garcia AKA, Lodi CR, Fonseca LF. O silêncio que permeia a sede do paciente cirúrgico: um relato 
de caso. In: Congresso Brasileiro de Enfermagem em Centro Cirúrgico, Recuperação Anestésica e Centro de Material e Esterilização, 13, 2017, São Paulo. Anais (on-line). São Paulo: SOBECC; 2017 [cited 2018 Dec 9]. Available from: http://sobecc. tmeventos.com.br/anais2017/pdfs/trabalho_2255. pdf

19. Silva RPJ, Rampazzo ARP, Nascimento LA, Fonseca LF. Desconfortos esperados e vivenciados por pacientes no pós-operatório imediato. Rev baiana enferm. 2018;32:e26070. DOI: http://dx.doi. org/10.18471/rbe.v32.26070

20. Landstrom M, Rehn I, Frisman GH. Perceptions of registered and enrolled nurses on thirst in mechanically ventilated adult patients in intensive care units - A phenomenographic study. Intensive Crit Care Nurs. 2009 Jun;25(3):133-9. DOI: 10.1016/j.iccn.2009.03.001

21. Aloamaka EO, Amabebe E, Ozoene JO, Obika LFO. Thirst perception, drinking, arginine vasopressin activity and associated neurohumoral factors. J Afri Assoc Physiol Sci [Internet]. 2018 [cited 2017 Oct 29];6(1):1-13. Available from: https://www.ajol.info/index.php/jaaps/article/ view/175955/165365
22. Campana MC, Fonseca LG, Melo Lopes DF, Martins PR. Percepção dos cuidadores quanto à sede da criança cirúrgica. Rev Rene. 2015 Dec;16(6):799808. DOI: $10.15253 / 2175-6783.2015000600005$

23. Pinheiro SL, Vasconcelos RO, Oliveira JLC, Matos FGOA, Tonini NS, Alves DCI. Taxa de cancelamento cirúrgico: indicador de qualidade em hospital universitário público. Reme - Rev Min Enferm. 2017 maio;21:e-1014. DOI: http://www. dx.doi.org/10.5935/1415-2762.20170024

24. Santos GAC, Bocchi SCM. Cancelamento de cirurgias eletivas em hospital público brasileiro: motivos e redução estimada. Rev Bras Enferm. 2017 May/June;70(3):561-8. DOI: http://dx.doi. org/10.1590/0034-7167-2016-0084

25. Nascimento LA, Fonseca LF, Santos CB. Interrater reliability testing of the Safety Protocol for Thirst Management. J Perianesth Nurs. 2018 Aug;33(4):527-36. DOI: 10.1016/j.jopan.2016.07.00 Recebido: 11 de novembro de 2019 Aprovado: 11 de fevereiro de 2020 Publicado: 2 de abril de 2020

A Revista Baiana de Enfermagem utiliza a Licença Creative Commons - Atribuição-NãoComercial 4.0 Internacional. https://creativecommons.org/licenses/by-nc/4.0/ Este artigo é de acesso aberto distribuído sob os termos da Licença Creative Commons (CC BY-NC). Esta licença permite que outros remixem, adaptem e criem a partir do seu trabalho para fins não comerciais. Embora os novos trabalhos tenham de lhe atribuir o devido crédito e não possam ser usados para fins comerciais, os usuários não têm de licenciar esses trabalhos derivados sob os mesmos termos. 